\title{
Keck spectroscopy and Spitzer space telescope analysis of the outer disk of the Triangulum spiral galaxy M 33
}

\author{
D. L. Block ${ }^{1,4}$, F. Combes $^{2}$, I. Puerari ${ }^{3}$, K. C. Freeman ${ }^{4}$, A. Stockton ${ }^{5}$, G. Canalizo ${ }^{6}$, T. H. Jarrett ${ }^{7}$, R. Groess ${ }^{1}$, \\ G. Worthey ${ }^{8}$, R. D. Gehrz ${ }^{9}$, C. E. Woodward ${ }^{9}$, E. F. Polomski ${ }^{9}$, and G. G. Fazio ${ }^{10}$ \\ ${ }^{1}$ School of Computational and Applied Mathematics, University of Witwatersrand, Private Bag 3, WITS 2050, South Africa \\ e-mail: block@cam.wits.ac.za \\ 2 Observatoire de Paris, LERMA, 61 Av. de l'Observatoire, 75014 Paris, France \\ 3 Instituto Nacional de Astrofísica, Optica y Electrónica, Calle Luis Enrique Erro 1, 72840 Tonantzintla, Puebla, México \\ ${ }^{4}$ Mount Stromlo and Siding Spring Observatories, Research School of Astronomy and Astrophysics, Australian National University, \\ Australia \\ 5 Institute for Astronomy, University of Hawaii, 2680 Woodlawn Drive, Honolulu, Hawaii, USA \\ 6 Institute of Geophysics and Planetary Physics and Department of Physics, University of California, Riverside, CA 92521, USA \\ 7 Infrared Processing and Analysis Center, 100-22, CALTECH, 770 South Wilson Ave, Pasadena, CA 91125, USA \\ 8 Washington State University, 1245 Webster Hall, Pullman, WA 99163-2814, USA \\ 9 Department of Astronomy, University of Minnesota, 116 Church St. SE, Minneapolis MN 55455, USA \\ 10 Harvard-Smithsonian Center for Astrophysics, 60 Garden St., Cambridge, MA 02138, USA
}

Received 26 June 2006 / Accepted 15 May 2007

\begin{abstract}
In an earlier study of the spiral galaxy M 33, we photometrically identified arcs or outer spiral arms of intermediate age (0.6-2 Gyr) carbon stars precisely at the commencement of the HI-warp. Stars in the arcs were unresolved, but were likely thermally-pulsing asymptotic giant branch carbon stars. Here we present Keck I spectroscopy of seven intrinsically bright and red target stars in the outer, northern arc in M33. The target stars have estimated visual magnitudes as faint as $V \sim 25^{m}$. Absorption bands of CN are seen in all seven spectra reported here, confirming their carbon star status. In addition, we present Keck II spectra of a small area 0.5 degree away from the centre of M 33; the target stars there are also identified as carbon stars. We also study the non-stellar PAH dust morphology of M 33 secured using IRAC on board the Spitzer Space Telescope. The Spitzer $8 \mu$ m image attests to a change of spiral phase at the start of the HI warp. The Keck spectra confirm that carbon stars may safely be identified on the basis of their red $J-K_{\mathrm{s}}$ colours in the outer, low metallicity disk of M33. We propose that the enhanced number of carbon stars in the outer arms are an indicator of recent star formation, fueled by gas accretion from the HI-warp reservoir.
\end{abstract}

Key words. galaxies: evolution - galaxies: spiral - galaxies: individual: M 33 (NGC598) - galaxies: Local Group galaxies: formation - galaxies: stellar content

\section{Introduction}

Like the two dominant spiral galaxies in the Local Group (MW and M 31), M33 is known to have a prominent warp. This warp is spectacular in the HI-21cm line: at many points the line of sight intersects the disk twice. In M 33 the warping commences at a radius of $\sim 5 \mathrm{kpc}$ and at a radius of $\sim 10 \mathrm{kpc}$ the rotation axis of neutral hydrogen gas is inclined by some 40 degrees to the axis of the inner disk, as revealed by the tilted ring model of Rogstad et al. (1976). More recently, Corbelli \& Schneider (1997) confirm that at a radius of $5 \mathrm{kpc}$ (about 3 disk scalelengths), the HI distribution in M 33 shows a distinct change in inclination.

Warps may be tidally generated when companions are obviously present; but in galaxies such as M 33 which do not have any close companions, warping and gas infall may be inextricably linked (for a review, see Sect. 7 in Binney 1992).

In this paper, we focus our attention on the outer warped disk of M33, and in particular on its red stellar population. If the HI-warp in M 33 is induced by the infall of gas, then one interesting confirmation would be the presence of a red intermediate age $(0.6 \mathrm{Gyr}-2 \mathrm{Gyr})$ population of carbon stars at the locale of the HI-warp. Indeed, C-stars are expected to be associated with recent star formation. Tsalmantza et al. (2006) have found them towards the center in the LMC and associated with spiral arms in M31, the loci of star formation. In the SMC and in M33, their sample size was too small to trace their radial distribution, but Rowe et al. (2005) found them also relatively more abundant in the outer parts of M 33 .

In an earlier study (Block et al. 2004), we presented nearinfrared images of M 33 from a deep subsample of 2MASS, in which individual stars were unresolved. The deep 2MASS images revealed remarkable arcs or spiral arms of red stars in the outer disk; the northern arc subtends $120^{\circ}$ in azimuth angle and $\sim 5^{\prime}$ in width (see Fig. 1). The northern arc is dominant although a very faint southern counterpart arc, forming a partial ring, can also be seen. The arcs lie at a radius of 2-3 disk scale lengths (in $V$, the disk scale length is 6 arcmin; Ferguson et al. 2006). Surprisingly, Fourier analysis of the deep 2MASS images showed that the dominant $m=2$ peak did not correspond to any inner spiral arm morphology seen in optical photographs, 




Fig. 1. A partial ring of very red stars is seen in this $J+H+K_{\mathrm{s}}$ image of M 33, secured from a special set of 2MASS observations wherein the integration time was increased by a factor of six. The ring is seen to full advantage with a simple ellipsoid model subtracted. Black areas are those of excess emission while white areas correspond to areas of deficit emission. The northern plume or arc spans up to $5^{\prime}$ in width and is located at a radius of 2 to 3 disk scale lengths (or 12 to 18 arcmin). An ellipse (of semi-major axis 16 arcmin) is drawn to pass through the arcs; North is up and and East to the left.

for which M 33 is so famous. Rather, the $m=2$ peak corresponds to the giant outer red arms in the 2MASS images (see Fig. 1).

Block et al. (2004) noted that the very red colour of the arcs could not be due to extinction by dust grains. Freedman et al. (1991) derive a mean value for the total colour excess for the Cepheids in $\mathrm{M} 33$ of $E(B-V)=0.10 \pm 0.09 \mathrm{mag}$, which includes both foreground (Milky Way) and internal M 33 extinction. The $K_{\mathrm{s}}$-band extinction $A\left(K_{\mathrm{s}}\right)$ is approximately one-tenth that in the optical (Rieke \& Lebofsky 1985), so the dust extinction at $K_{\mathrm{s}}$ is only $\sim 0.03 \mathrm{mag}$. Extinction by dust grains cannot generate the very red colours found in the arcs in the outer disk of M 33, where the extinction at $K_{\mathrm{s}}$ would be even smaller.

Our methodology differs from conventional ways of photometrically identifying carbon stars using intermediate band filters $(V, I, 77,81)$ as presented by Rowe et al. (2005) for M 33, using the Canada-France-Hawaii Telescope (CFHT). With such a method, one needs a large enough telescope to resolve individual stars. The $(V, I, 77,81)$ photometric method was pioneered by Cook et al. (1986); another viable set of filters to identify carbon stars are the Sloan filters. Demers \& Battinelli (2005) used the three Sloan filters $g, r, i$, but again resolution of stars is crucial. Demers \& Battinelli (2005) also used the CFHT with the Megacam camera to identify 361 new C-star candidates in M 31.
Some investigators (e.g. Tsalmantza et al. 2006) have focussed their attention on identifying carbon stars on the basis of the red $J-K_{\mathrm{s}}$ tail (see Figs. 1, 4, 6 and 9 in Tsalmantza et al. 2006). The usefulness of such a methodology is that nearinfrared all sky surveys secured with moderate sized telescopes can potentially be exploited to identify carbon stars in spiral galaxies well outside our Local Group, where individual stars are not resolved.

The arcs seen in Fig. 1 are identified on the basis of their red $J-K_{\mathrm{s}}$ colours. They lie around 15 arcmin in radius, between 12 and 18 arcmin, or 2-3 disk scale lengths, whereas the actual disk truncates at 5 scale lengths (Ferguson et al. 2006). They are therefore like spiral arms in the outer disk, as seen also by Rowe et al. (2005). The latter authors have identified the $\mathrm{C}$ and M-type stars in the AGB population, and traced the carbon star to $\mathrm{M}$ star ratio $(\mathrm{C} / \mathrm{M})$ as a function of radius. This ratio is not only an index of age but also of metallicity, increasing in metal-poor regions. Indeed, a $\mathrm{C}$-star requires that the $\mathrm{O}$-dominated surface be reversed to C-dominated, due to the dredged-up material from the star interior. This is easier to do when the surface is metalpoor. Rowe et al. (2005) show that the C/M ratio increases with radius and then flattens beyond 20 arcmin (or $5 \mathrm{kpc}$ ), indicating 


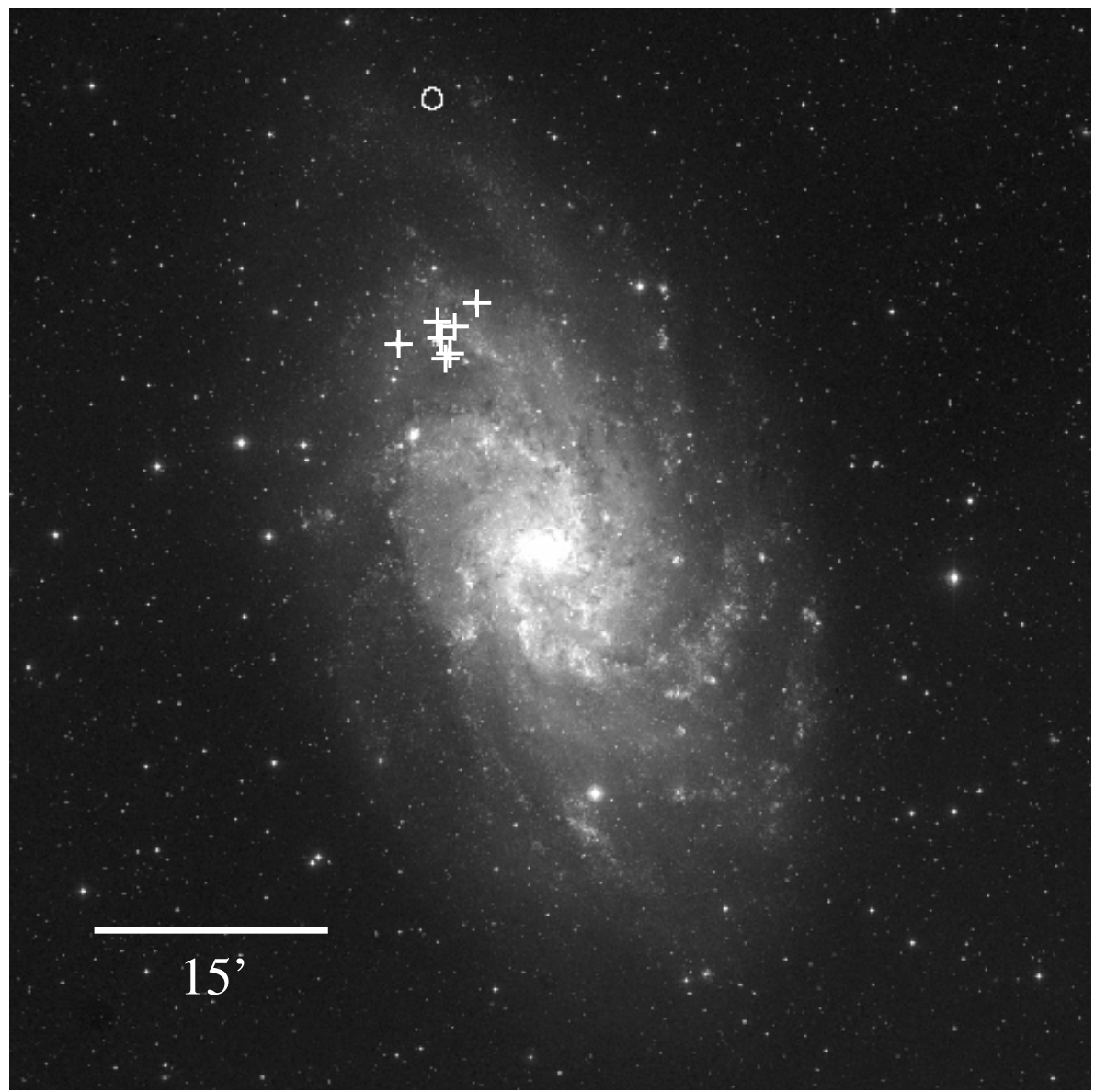

Fig. 2. The positions of the seven stars listed in Table 1 are indicated by plus signs; the stars lie in the northern swath of carbon bearing stars identified photometrically in an earlier study (Block et al. 2004). Spectra of these stars (see Fig. 3) were secured using the Keck I telescope in Hawaii. Also observed with its twin sister telescope, Keck II, are two stars which lie 0.5 degrees away from the centre of M 33 in an area identified by a white circle. These outlying stars are representative of a very red family identified in near-infrared imaging with the Hale $5 \mathrm{~m}$ reflector at Mount Palomar. In this DSS image, North is up and East is to the left.

a metallicity gradient covering most of the disk, including the outer spiral arcs.

Our $J H K_{\mathrm{s}}$ photometric method uses the fact that the colour of the northern arc extended to very red colours of $J-K_{\mathrm{s}}>1.1$. It was argued that while very old $\mathrm{M}$ giants of solar abundance can indeed reach $J-K_{\mathrm{s}} \sim 1$ (see e.g. Fig. 2 in Bessell \& Brett 1998 ) and even redder if they are super-metal-rich (see Frogel \& Whitford 1987), stars with $J-K_{\mathrm{s}}>1$ in low-metallicity regions cannot be M-giants but rather, very red carbon stars. There is a strong radial metallicity gradient in M $33(-0.16 \mathrm{dex} / \mathrm{kpc}$ in $\mathrm{O} / \mathrm{H}$, over 4-5 kpc, see Beaulieu et al. 2006). Beaulieu et al. have confirmed this metallicity gradient, already found in HII regions, B-supergiants or planetary nebulae, by detecting beat Cepheids in M33. An excellent discussion of the metallicity gradient in M 33 may be found in Magrini et al. (2007). The outer regions of M33 are relatively metal-poor, and solar abundance is reached only in the very central domain of M33.

In this paper, we examine the robustness of our photometric technique as a stepping stone to exploring the outer disks of more distant spiral galaxies, wherein individual carbon stars may be present, but unresolved. To this end, we need follow-up spectroscopy. Here we discuss exploratory Keck I and Keck II spectroscopic observations of a few of the reddest and intrinsically brightest stars in the northern arc. All seven of our target candidates have very red $J-K_{\mathrm{s}}$ colours and should be confirmed to be TP-AGB carbon stars if our methodology is correct.

\section{Observations}

As a prelude to conducting the Keck spectroscopy, we imaged a section of the northern plume of M 33 with the Hale 5 m reflector
Table 1. Magnitudes and colors of our target stars in the northern red arc of M 33 .

\begin{tabular}{cccccccc}
\hline \hline Star & $J$ & $H$ & $K_{\mathrm{s}}$ & $J-K_{\mathrm{s}}$ & $J-H$ & $H-K_{\mathrm{s}}$ & $M_{K_{\mathrm{s}}}$ \\
\hline 1 & 17.96 & 16.76 & 15.91 & 2.05 & 1.20 & 0.85 & -8.74 \\
2 & 18.49 & 17.09 & 16.32 & 2.16 & 1.40 & 0.77 & -8.33 \\
3 & 19.61 & 18.04 & 16.66 & 2.96 & 1.57 & 1.39 & -7.99 \\
4 & 18.57 & 17.26 & 16.49 & 2.08 & 1.31 & 0.77 & -8.16 \\
5 & 18.82 & 17.35 & 16.54 & 2.28 & 1.47 & 0.81 & -8.11 \\
6 & 18.42 & 17.24 & 16.46 & 1.97 & 1.18 & 0.78 & -8.19 \\
7 & 18.37 & 16.91 & 15.82 & 2.55 & 1.46 & 1.10 & -8.83 \\
\hline
\end{tabular}

at Mount Palomar. We used the $2048 \times 2048$ array near-infrared camera WIRC (Wilson et al. 2003), to resolve individual stars. The field of view is $8.5^{\prime} \times 8.5^{\prime}$ with 0.25 arcsec pixels. The seeing $F W H M$ is $0.8^{\prime \prime}$ in $J$, and $0.7^{\prime \prime}$ in the $K_{\mathrm{s}}$ band. The telescope was centered at $01 \mathrm{~h} 34 \mathrm{~m} 28.1 \mathrm{~s},+30 \mathrm{~d} 54 \mathrm{~m} 00 \mathrm{~s}(\mathrm{~J} 2000)$. The total $J H K_{\mathrm{s}}$ integration time was $\sim 9$ min, reaching a limiting surface

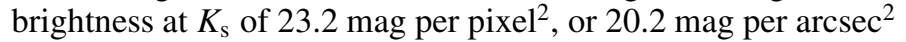
$(4 \times 4$ pixels gives 1 square arsec). The point source photometry $S / N=10$ limits are 19.0, 18.0, 16.9 mag in $J H K_{\mathrm{s}}$, respectively.

Table 1 lists the magnitudes and colours of 7 candidate targets in the outer northern arc seen in Block et al. (2004). Their positions are indicated by plus signs (+) in Fig. 2. In deriving the absolute magnitudes in Table 1, we assume a distance modulus to M 33 of $24.64^{m}$, corresponding to a linear distance of $840 \mathrm{kpc}$ (Freedman et al. 1991). Noting that $E(B-V)=0.10 \pm 0.09 \mathrm{mag}$ (Freedman et al. 1991), and that the $K_{\mathrm{s}}$-band extinction is approximately one-tenth that in the optical (Rieke \& Lebofsky 1985), we use a dust extinction correction for the $K_{\mathrm{s}}$ apparent magnitudes of $0.03 \mathrm{mag}$. 
Spectra of these carbon star candidates were obtained on 2004 Aug. 17 UT with the Low Resolution Imaging Spectrograph (LRIS; Oke et al. 1995) attached to the Keck I telescope. The aperture mask slitlets had widths and lengths corresponding to $1^{\prime \prime} .2$ and $11^{\prime \prime} .5$, respectively. The 600 groove $\mathrm{mm}^{-1}$ grating gave a resolution of $\sim 8 \AA$ per projected slit width and a spectral range from 6825 to $9415 \AA$ for slitlets near the centre of the mask. The stars were dithered along the slitlets between the 2 exposures, each $600 \mathrm{~s}$ long. The spectrum from each slitlet was extracted and reduced independently, the wavelength calibration being derived from airglow lines. An approximate flux calibration was produced by observing the spectrophotometric standard star G191B2B (Massey et al. 1988) in one of the slitlets near the centre of the mask and transfering the system response function found for this slitlet to the same wavelength intervals for the other slitlets. This approach neglects any differential vignetting or other field-dependent variations in response, but these are expected to be small for LRIS in any case.

Also secured with the Keck II telescope were spectroscopic observations of an area 0.5 degree away from the centre of M 33 (circled in Fig. 2). Near-infrared imaging with the Hale $5 \mathrm{~m}$ reflector at Palomar had revealed stars with very red $J-K_{\mathrm{s}}$ colours in this outer low metallicity region. The data were obtained on 2004 July 18 UT using the Echellette Spectrograph and Imager (ESI; Sheinis et al. 2002) on the Keck II telescope. We used the echellete mode which allows a wavelength coverage from 3900 to $11060 \AA$ and yields a dispersion of $\sim 56 \mathrm{~km} \mathrm{~s}^{-1}$ with the 1 " slit. The spectra were obtained at parallactic angle near transit, and the total exposure was $1200 \mathrm{~s}$ for each object.

The spectra were reduced using a combination of J. Prochaska's IDL reduction package, ESIRedux, and the echelle tasks in IRAF. The spectra were flux calibrated using spectrophotometric standards from Massey et al. (1988) also observed with the slit at the parallactic angle.

Observations of M33 were also made using all four bands of the Infrared Array Camera (IRAC) mounted on the Spitzer Space Telescope (Werner et al. 2004; Gehrz et al. 2007) on 2005 January 21 as part of a Spitzer Guaranteed Time Observing Program (Program ID 5) conducted by Spitzer Science Working Group member R. D. Gehrz.

The IRAC instrument (Fazio et al. 2004) is composed of four detectors that operate at $3.6 \mu \mathrm{m}$ (channel 1), $4.5 \mu \mathrm{m}$ (channel 2), $5.8 \mu \mathrm{m}$ (channel 3) and $8.0 \mu \mathrm{m}$ (channel 4). All four detector arrays are $256 \times 256$ pixels in size with mean pixel scales of 1.221 , $1.213,1.222$, and $1.220^{\prime \prime}$ pixel $^{-1}$ respectively. The IRAC filter band centers are at 3.548, 4.492, 5.661 and $7.87 \mu \mathrm{m}$ and the adopted zero magnitude fluxes are $280.9 \mathrm{Jy}$ (channel 1), $179.7 \mathrm{Jy}$ (channel 2), 115.0 Jy (channel 3) and 64.1 Jy (channel 4) as described in the IRAC Data Handbook V3.0 (see Gehrz et al. 2007).

The M33 mapping sequence consisted of 438 frames per channel, including a 3 point $1 / 2$ pixel dither for each map position. The integration time was $12 \mathrm{~s}$ per frame.

The raw Spitzer data were processed and flux calibrated with version 11.0.2 of the Spitzer Science Center (SSC) pipeline. Post-BCD processing was carried out using an artifact mitigation algorithm developed by Carey (2005) and the 2005 September 30 Linux version of the SSC MOPEX software (Makovoz \& Khan 2005). The artifact mitigation algorithm alleviates the effects of muxbleed, column pulldown/pullup, electronic banding, and bias variations between the images. Three additional corrections were implemented with MOPEX: Background Matching, Outlier Detection, and
Mosaicing. Background matching was performed by minimizing the pixel differences in overlapping areas with respect to a constant offset computed by the program. Cosmic rays and other outliers were detected and eliminated with the Outlier Detection module. In the final step the images were reinterpolated to a pixel scale of approximately $1.224^{\prime \prime}$ pixel $^{-1}$ and mosaiced to create a final image spanning approximately $1.2^{\circ} \times 1.4^{\circ}$.

\section{Analysis of the Keck I and Keck II spectra}

Even for nearby M33, identification of carbon stars by spectroscopic means necessitates the use of the largest of groundbased telescopes. The first carbon star to be spectroscopically confirmed in M 33 dates back to the work of Mould \& Aaronson (1986; Fig. 7) who used the Hale $5 \mathrm{~m}$ reflector at Palomar. The individual $V$ magnitudes of each target star in Table 1 are estimated, from their $J H K_{\mathrm{s}}$ colours, to be fainter than $22^{m}$, with one star (star 3 in Fig. 3) as faint as $V \sim 25^{m}$. The spectra sample only the bright end of the $K_{\mathrm{s}}$-band luminosity function for carbon stars, which ranges from $M_{\mathrm{K}} \sim-6$ to -9 . Much longer integration times would be needed for intrinsically fainter carbon stars.

Carbon stars show a plethora of molecular spectral features, including the $\mathrm{C}_{2}$ Swan bands and the $\mathrm{CN}$ bands. The presence of the hugely dominant CN bands shortward of $7000 \AA$ and $8000 \AA$ and longward of $9000 \AA$ are indicated by tick marks in Fig. 3 and unambiguously reveal the C-star status for each of these seven stars.

\section{Analysis of the ISM dust morphology in M 33}

The carbon star arcs are clearly seen in $J H K_{\mathrm{s}}$ images, but the global ISM dust morphology can also be effectively traced at $8 \mu \mathrm{m}$. The reason is as follows: as carbon stars pulsate, their atmospheres become extended and matter may reach a temperature low enough for some elements to condense into very small particles ("star dust"). An outflow develops as a result of radiation pressure and the star is progressively surrounded by an expanding circumstellar shell of dust and gas (Le Bertre et al. 2003). Emission from the shells of both carbon stars and also dust from O-rich shells is detected at $8 \mu \mathrm{m}$ (Buchanan et al. 2006). Buchanan and co-workers note that "most of the C-rich stars have spectra that are dominated by warm dust" and these authors show that stars such as MSXLMC587 (O-rich) and MSXLMC1488 (C-rich) both show emission in the IRAC $8 \mu \mathrm{m}$ window (see their Fig. 2, for example). The trend is for the flux to climb from longer $(\sim 38 \mu \mathrm{m})$ to the shorter wavelength of $8 \mu \mathrm{m}$. As an interesting aside, one does not need high ultraviolet excitation to produce infrared emission features: stars as cool as $3000 \mathrm{~K}$ can generate PAH features, for example, as discussed by Li and Draine (2002).

Carbon stars are one of the most important contributors to the replenishment of dust in the interstellar medium. Our IRAC image of M33 at wavelength $8 \mu \mathrm{m}$ offers unprecendented insight into the global ISM dust morphology. Deprojection of our IRAC images of M 33 were facilitated by adopting $\log R_{25}=$ 0.23 (RC3) where $R$ is the ratio of the major to minor axis, and a position angle of 23 degrees (Deul \& van der Hulst 1987; Regan \& Vogel 1994). In order to remove the small contribution of starlight (at the end of the Rayleigh-Jeans tail) from the $8 \mu \mathrm{m}$ image, we subtract a scaled version of the IRAC $3.6 \mu \mathrm{m}$ map. As in Block et al. (2006), we generate a "non-stellar" pure dust map from $8 \mu \mathrm{m}-\kappa 3.6 \mu \mathrm{m}$, where $\kappa$ (a constant) assumes the value 

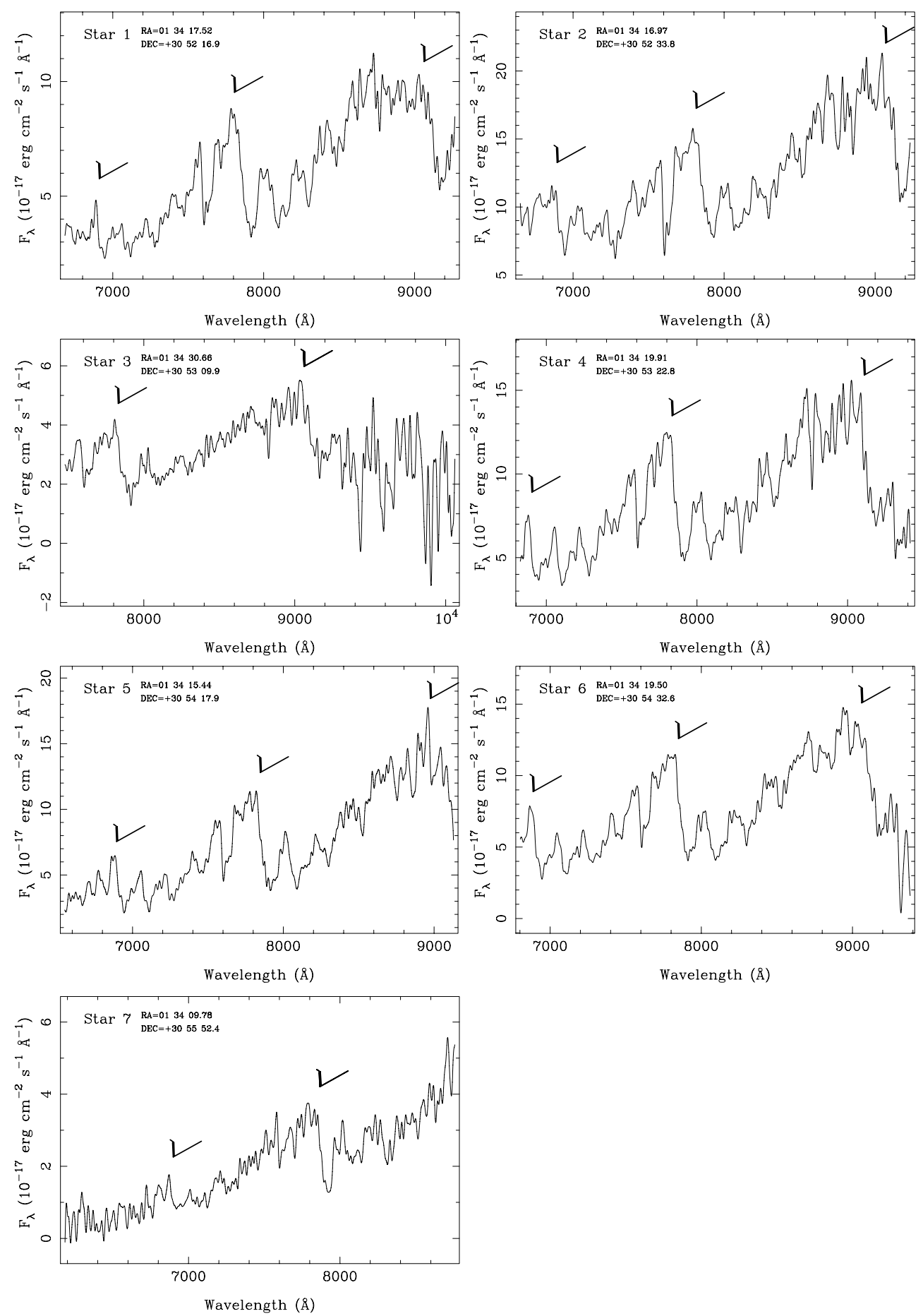

Fig. 3. Spectra of the seven stars seen in Fig. 2, secured using the Keck I telescope. All C stars candidates are very red, with $J-K_{\mathrm{s}}>2$ mag; their individual colors are listed in Table 1 . The coordinates of each star are indicated in each panel. Tick marks identify the prominent $\mathrm{CN}$ bands found in each star, shortward of $7000 \AA$ and $8000 \AA$ and longward of $9000 \AA$. Each star is spectroscopically confirmed to belong to the thermally-pulsing asymptotic giant branch carbon star family.

of 0.25 . This subtraction removes the contribution from stellar photospheres and leaves only the emission from dust grains, which trace the ISM morphology. The major uncertainties entering into the precise value of $\kappa$ are, firstly, the extended aperture correction, and secondly, the exact mid-IR colour of the stellar populations which one is modeling by scaling the $3.6 \mu \mathrm{m}$ emission. The second correction introduces uncertainties of order $0.05 \mathrm{mag}$ or perhaps larger (Ashby, private communication). A slightly smaller value of $\kappa(0.232$ instead of 0.25$)$ has been suggested by Helou et al. (2004), but our use of a slightly higher photospheric contribution (at a level of 2 percent) is well within the correction uncertainties; the choice of $\kappa$ is only accurate at the 10-15 percent (and not one or two percent) level.

A complimentary method could, of course, be to focus our attention on photospheric emission, and not ISM dust emission. At the shorter IRAC wavelength of $3.6 \mu \mathrm{m}$, carbon star photospheres would be detected, but so would the photospheres of O-rich stars. A full presentation and analysis of all IRAC images 

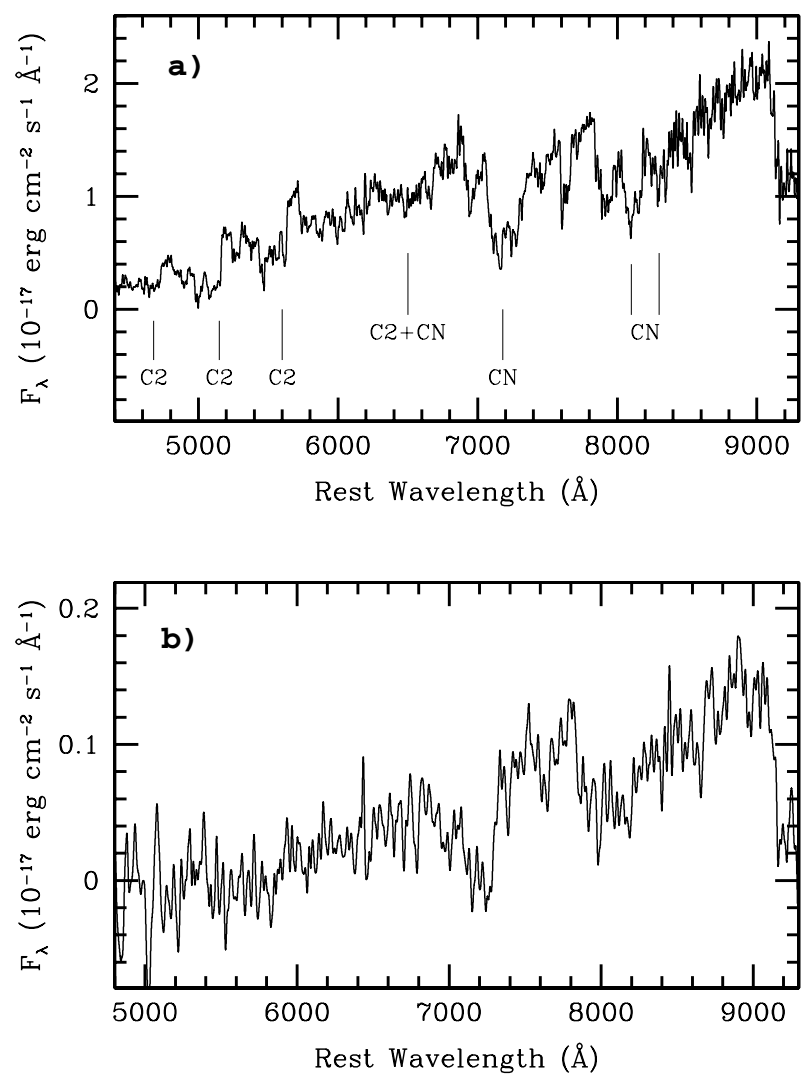

Fig. 4. Spectra with the Keck II telescope, of two stars 0.5 degree ( $\sim 5$ scalelengths) away from the centre of M33 (the field circled in Fig. 2), centered at approximately $\mathrm{RA}(2000)=1 \mathrm{~h} 34 \mathrm{~m} 21 \mathrm{~s}$ and $\operatorname{Dec}(2000)=31^{\circ} 08^{\prime} 45^{\prime \prime} \cdot 7$. The dominant $\mathrm{CN}$ absorption features are clearly evident and betray the signature of TP-AGB carbon stars.

of M 33 will be presented elsewhere (Gehrz et al., in preparation). In this paper, we confine our attention to the dust grain morphology, as we did for the Andromeda Spiral in Block et al. (2006).

The Fourier method has been extensively discussed in a number of papers (e.g., Considère \& Athanassoula 1982; Puerari \& Dottori 1992, among others). In the Fourier method, an image is decomposed into a basis of logarithmic spirals of the form $r=r_{o} \exp \left(-\frac{m}{p} \theta\right)$. The Fourier coefficients $A(p, m)$ can be written as

$A(p, m)=\frac{1}{D} \int_{-\pi}^{+\pi} \int_{-\infty}^{+\infty} I(u, \theta) \exp [-\mathrm{i}(m \theta+p u)] \mathrm{d} u \mathrm{~d} \theta$.

Here, $u \equiv \ln r, r$ and $\theta$ are the polar coordinates, $m$ represents the number of the arms, $p$ is related to the pitch angle $P$ of the spiral by $P=\operatorname{atan}(-m / p)$, and $I(u, \theta)$ is the distribution of light of a given deprojected galaxy, in a $(\ln r, \theta)$ plane. $D$ is a normalization factor written as

$D=\int_{-\pi}^{+\pi} \int_{-\infty}^{+\infty} I(u, \theta) \mathrm{d} u \mathrm{~d} \theta$.

In practice, the integrals in $u \equiv \ln r$ are calculated from a minimum radius (selected to exclude the bulge where there is no information of the arms) to a maximum radius (which extends to the outer limits of the arms in our images).

The inverse Fourier transform can be written as

$S(u, \theta)=\sum_{m} S_{m}(u) \mathrm{e}^{\mathrm{i} m \theta}$

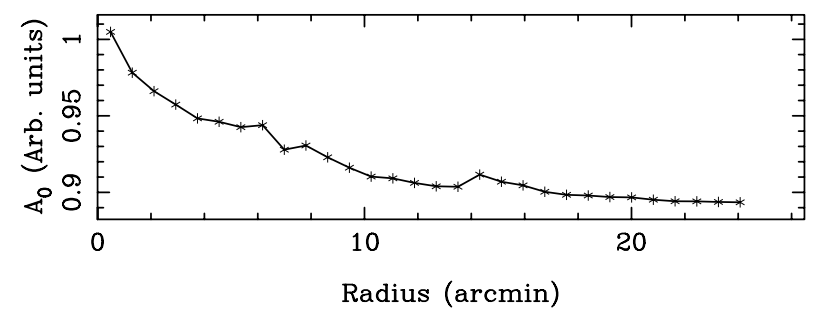

$M=2$

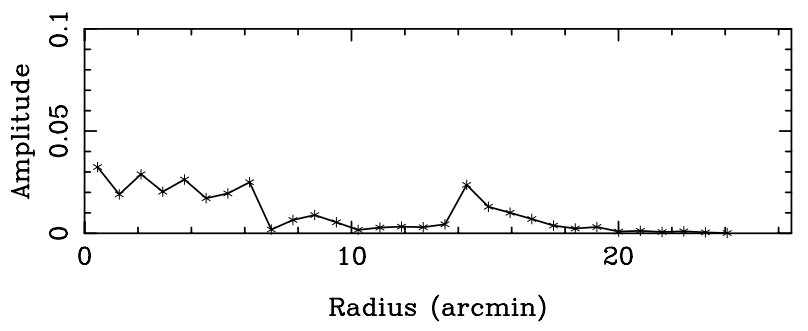

$M=2$

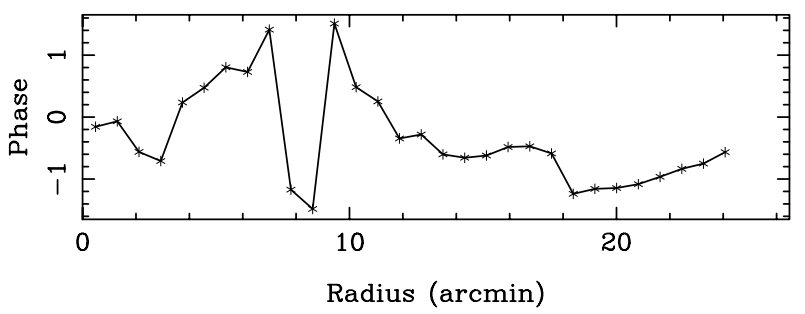

Fig. 5. The topmost panel shows a dust emissivity $8 \mu \mathrm{m}$ radial profile. In the middle panel is shown the amplitude of the Fourier $m=2$ mode as a function of radius. The local peak at $r=14^{\prime}$ coincides with the inner radial limit of the arcs. The outer spiral arms or "arcs" correspond to the higher $m=2$ amplitude between 14 and 18 arcmin. The phase of the $m=2$ component is presented in the lower panel. We see a change of phase in the dust emissivity distribution at 18 arcmin, at the outer boundary of the outer spirals, precisely the same radius where Corbelli \& Schneider (1997) find a warp in HI.

where

$S_{m}(u)=\frac{D}{\mathrm{e}^{2 u} 4 \pi^{2}} \int_{p_{-}}^{p_{+}} G_{m}(p) A(p, m) \mathrm{e}^{\mathrm{i} p u} \mathrm{~d} p$

and $G_{m}(p)$ is a high frequency filter used to smooth the $A(p, m)$ spectra at the interval ends (see Puerari \& Dottori 1992), and it has the form

$G_{m}(p)=\exp \left[-\frac{1}{2}\left(\frac{p-p_{\max }^{m}}{25}\right)^{2}\right]$

where $p_{\max }^{m}$ is the value of $p$ for which the amplitude of the Fourier coefficients for a given $m$ is maximum. The chosen interval ends $\left(p_{+}=+50\right.$ and $\left.p_{-}=-50\right)$, as well as the step-size $\mathrm{d} p=0.25$, are suitable for the analysis of galactic spiral arms.

Firstly, the Fourier $m=2$ mode in the $8 \mu \mathrm{m}$ image also does not correspond to the inner spiral arm morphology, but rather shows a local peak at a radius of 14', just longward of 2 scalelengths. This is precisely where the outer red arcs were detected by Block et al. (2004).

Another intriguing fact is that there is a distinct change of phase in the ISM dust morphology at 3 disk scale-lengths $\left(18^{\prime}\right)$, which is precisely the region of the outer boundaries of the "arcs" or outer spiral arms, where Corbelli \& Schneider (1997) find the prominent HI-warp. 
We believe it fully consistent to propose that the very red, and relatively metal-poor stars have recently been formed by gas infall which is inextricably tied to its strong HI-warp. It seems highly plausible that fresh low-metallicity gas is being fed to the host galaxy M 33. The outer disk from which mass is accreted is inclined to the inner disk of M 33, and has a different angular momentum, as revealed by the tilted disk model.

Our conclusions are compatible with the work of Rowe et al. (2005) who published a spatial map of the C/M-star ratio, that reveals conspicuous maxima at exactly the positions of what we call "arcs", or outer spiral arms. They interpret the radial distribution of the $\mathrm{C} / \mathrm{M}$ ratio uniquely as a metallicity indicator, and conclude that there is a strong metallicity gradient up to $5 \mathrm{kpc}$ (20 arcmin) and then the gradient flattens out. Their interpretation is in terms of the viscous disk model, where the outer shear of the rotation curve produces mixing in the outer parts, able to wash out abundance gradients.

The viscous disk model certainly applies to M33 to some extent, and is certainly a needed ingredient of the external gas accretion model. The flattening of the metallicity gradient could be linked to the accretion of low-metallicity gas. The viscous accretion disk model has been frequently invoked to build exponential galaxy disks, either with viscous torques to exchange angular momentum, or with gravitational torques due to spiral arms or bars. In the latter case the equivalent viscosity is called "gravitational viscosity" (e.g. Lin \& Pringle 1987). If the viscous and star formation time scales are of the same order, then the resulting stellar disk has an exponential distribution independent of the disk rotation law and of the assumed viscosity prescription. The expected metallicity gradient is also exponential, as computed by Tsujimoto et al. (1995). As for M 33, it is highly possible that the "viscous disk" model applies until a radius of $5 \mathrm{kpc}$ (20 arcmin), i.e. just outside the arcs, and is the mechanism with which the accreted gas coming from the warped component is consumed into stars and disk formation. The optical disk is exponential up to this radius.

\section{Conclusion}

We have confirmed, through Keck spectroscopy of individual stars, the presence of an enhanced abundance of carbon stars in the outer spiral arms in M33, in particular in the two "arcs" identified previously from their red colour (Block et al. 2004). Our conclusions are in very good agreement with the recent work of Rowe et al. (2005), who have produced a spatial map of the $\mathrm{C} / \mathrm{M}$-star ratio in M 33 showing the conspicuous outer arms. We also see the outer spiral arms in the Fourier $m=2$ component of the Spitzer $8 \mu \mathrm{m}$ dust emission image. These arms correspond to the radius where the HI- $21 \mathrm{~cm}$ warp starts, and we propose an interpretation in terms of recent star formation, fueled from the gas accreted from this outer reservoir. A very similar conclusion, in terms of M 33 accreting gas, has recently been reached by Magrini et al. (2007), on the basis of $\mathrm{O} / \mathrm{H}, \mathrm{S} / \mathrm{H}$ and $[\mathrm{Fe} / \mathrm{H}]$ abundances in the Triangulum Spiral Galaxy M33.

The HI gas reservoirs in the outer parts of galaxies are observed outside nearly all spiral disks (Sancisi 1983). Their conspicuous warped morphology, even in the absence of any perturbation or companion, implies that this gas is being almost continuously accreted, with a different angular momentum than that of the inner disk (e.g. Binney 1992). Keres et al. (2005) discuss cold gas accretion along filaments in the cosmic web. Accreting systems in gas need not show any signs of accretion in stars, such as the presence of tidal tails, stellar loops or close companions.
The implications of the presence of carbon stars in the outer disk of M 33 immediately beckons the question of a possible ubiquity of such an intermediate age population in the outer domains of other spiral disks out to the Virgo cluster and beyond.

At hand is the potential to apply our photometric technique of identifying thermally-pulsing asymptotic giant branch carbon stars to more distant spirals on the basis of their red $J-K_{\mathrm{s}}$ colours in low metallicity domains, an approach adopted by other teams such as Tsalmantza et al. (2006). It is a sobering thought that it takes a groundbased class 8-10 m telescope to secure individual carbon star spectra in the outer disk of M 33, our second closest spiral, in realistic amounts of observing time. Hence the urgent need to identify unresolved sets of carbon stars in distant spiral galaxies, on the basis of colours alone - without resort to resolving such stars first with thirty metre class telescopes, and then imaging them through Sloan or $(V, I, 77,81)$ filters.

Carbon stars may make an important contribution to the IR luminosity of high-redshift galaxies. Using the Large Magellanic Cloud as a guide, carbon stars are produced in large numbers between ages of about 0.6 and 2 Gyr. Therefore galaxies that undergo a burst of star formation will have their IR light boosted 0.6 Gyr later, and this extra light will die away after about 2 Gyr. We know that star formation in the early universe (Bouwens et al. 2003) was already proceeding at redshift of at least $z \sim 6$. There may be an epoch starting about 0.6 Gyr after the onset of star formation in the universe (i.e. at redshifts $z \sim 4$ ) characterized by large numbers of carbon stars. The dominant output from the carbon stars will be redshifted into the mid-infrared where these stars could double the observed extragalactic flux. Maraston (2005) indeed proposes the use of carbon stars as an age indicator for high-redshift stellar populations. Instruments like the Mid-Infrared Instrument on board the JWST are needed to image such galaxies in their (rest-frame) $2.2 \mathrm{mi}-$ cron band.

Acknowledgements. This paper was completed whilst DLB was a Visiting Professor at the Mount Stromlo and Siding Spring Observatories, Canberra; the hospitality of Ken and Margaret Freeman is very warmly acknowledged. D.L.B. and I.P. are indebted to the Anglo American Chairman's Fund, Mr. C. Sunter, Mrs. M. Keeton and the Board of Trustees. I.P. acknowledges support from the Mexican foundation CONACyT under project 35947-E. This paper is based in part on data obtained at the W. M. Keck Observatory, which is operated as a scientific partnership among the California Institute of Technology, the University of California and the National Aeronautics and Space Administration. The Observatory was made possible by the financial support of the W. M. Keck Foundation. This work is also partially based on observations made with the Spitzer Space Telescope, which is operated by the Jet Propulsion Laboratory, California Institute of Technology under a contract with NASA. R.D.G. and C.E.W. were supported by NASA through an award issued by JPL/Caltech. D.L.B. warmly thanks the Vice-Chancellor of the University of the Witwatersrand for the Vice-Chancellor's Research Award in 2006. D.L.B. also expresses much gratitude to Fani Titi of the TISO Foundation for his stellar support. We thank M. Ashby, P. Wood and S.P. Willner for their input. Finally, we thank the anonymous referee for insightful comments.

\section{References}

Beaulieu, J.-P., Buchler, J., Marquette, J.-B., et al. 2006, ApJ, 653, L101 Bessell, M. S., \& Brett, J. M. 1998, PASP, 100, 1134

Binney, J. 1992, ARAA, 30, 51

Block, D. L., Freeman, K. C., Jarrett, T. H., et al. 2004, A\&A, 425, L37 Block, D. L., Bournaud, F., Combes, F., et al. 2006, Nature, 443, 832 Bouwens, R. J., Illingworth, G. D., Rosati, P., et al. 2003, ApJ, 595, 589 Buchanan, C. L., Kastner, J. H., Forresst, W. J., et al. 2006, AJ, 132, 1890 Carey, S. 2005, http://spider.ipac.caltech.edu/staff/carey/ irac_artifacts/ 
Considere, S., \& Athanassoula, E. 1982, A\&A, 111, 28

Cook, K. H., Aaronson, M., \& Norris, J. 1986, ApJ, 305, 634

Corbelli, E., \& Schneider, S. E. 1997, ApJ, 479, 244

Demers, S., \& Battinelli, P. 2005, A\&A, 436, 91

Deul, E. R., \& van der Hulst, J. M. 1987, A\&AS, 67, 509

Fazio, G. G., Hora, J. L., Allen, L. E., et al. 2004, ApJS, 154, 10

Ferguson, A., Irwin, M., Chapman, S., et al. 2006 [arXiv : astro-ph/0601121]

Freedman, W. L., Wilson, C. D., \& Madore, B. F. 1991, ApJ, 372, 455

Frogel, J. A., \& Whitford, A. E. 1987, ApJ, 320, 199

Gehrz, R. D., et al. 2007, Rev. Sci. Instrum. 78, 011302

Helou, G., Roussel, H., Appleton, P., et al. 2004, ApJS, 154, 253

Keres, D., Katz, N., Weinberg, D. H., \& Davé, R. 2005, MNRAS, 363, 2

Le Bertre, T., Tanaka, M., Yamamura, I., \& Murakami, H. 2003, A\&A, 403, 943

Li, A., \& Draine, B. T. 2002, ApJ, 572, 232

Lin, D. N. C., \& Pringle, J. E. 1987, MNRAS, 225, 607

Magrini, L., Corbelli, E., \& Galli, D. 2007, A\&A, 470, 843

Makavoz, D., \& Khan, I. 2005, in Mosaicing with MOPEX Astronomical Data Analysis Software and Systems XIV, ASP Conf. Ser., ed. P. L. Shopbell, M. C. Britton, \& R. Ebert, in press
Maraston, C. 2005, in Multiwavelength mapping of galaxy formation and evolution, ed A. Renzini, \& R. Bender (Berlin: Springer), 290

Massey, P., Strobel, K., Barnes, J. V., \& Anderson, E. 1988, ApJ, 328, 315

Mould, J., \& Aaronson, M. 1986, ApJ, 303, 10

Oke, J. B., Cohen, J. G., Carr, M., et al. 1995, PASP, 107, 375

Puerari, I., \& Dottori, H. A. 1992, A\&AS, 93, 469

Regan, M. W., \& Vogel, S. N. 1994, ApJ, 434, 536

Rieke, G. H., \& Lebofsky, M. J. 1985, ApJ, 288, 618

Rogstad, D. H., Wright, M. C. H., \& Lockhart, I. A. 1976, ApJ, 204, 703

Rowe, J. F., Richer, H. B., Brewer, J. P., \& Crabtree, D. R. 2005, AJ, 129, 729

Sancisi, R. 1983, Internal Kinematics and Dynamics of Galaxies, IAU Symp., 100,55

Sheinis, A. I., Bolte, M., Epps, H. W., et al. 2002, PASP, 114, 851

Tsalmantza, P., Kontizas, E., Cambrésy, L., et al. 2006, A\&A, 447, 89

Tsujimoto, T., Yoshii, Y., Nomoto, K., \& Shigeyama, T. 1995, A\&A, 302, 704

Werner, M. W., Roelling, T. L., Low, F. J., et al. 2004, ApJS, 154, 1

Wilson, C. D. 1991, AJ, 101, 1663

Wilson, J. C., Eikenberry, S. S., Henderson, C. P., et al. 2003, Proc. SPIE, 4841, 451 